\title{
THE CAVENDISH LECTURE
}

ON

\section{THE ETIOLOGY AND DIAGNOSIS OF CEREBRO-SPINAL FEVER.}

Read before the West London Medico-Chirurgical Society. By WILLIAM OSLER, M.D., F.R.S., F.R.C.P., Professor of Medicine, Johns Hopkins University, Baltimore.

Is practice we occasionally meet with a meningitis which is not tuberculous, which is not a complication of pneumonia or ulcerative endocarditis, and which is not a sequence of ear disease or of injury, and which is not a terminal infection in some chronic malady. As the meninges of both brain and cord are diffusely inflamed, we label the condition cerebrospinal meningitis. At intervals, in certain regions, the cases multiply, and we then speak of epidemic cerebro-spinal fever. Until recently my experience, though somewhat varied, had been confined to a few instances in which the absence of the usual factors justified the diagnosis of the sporadic form of this disease. Within the past year a small outbreak in Baltimore has enabled me to study certain points in the etiology and diagnosis of this most interesting affection.

The Features of Cerebro-Spinal Fever as an EPIDEMIC.

Upon these I shall only touch very briefly.

(a) One of the most fatal of all acute diseases, it fortunately takes a very humble position among epidemics as a destroyer. No fever attacks so few individuals in a community during its periods of prevalence; so that the general mortality may be but slightly increased. On the other hand, scarcely any known fever kills so large a proportion of those attacked. The recent epidemic in Boston illustrates this very well of I I cases in three hospitals 76 died, a mortality of 68.5 per cent.

(b) The outbreaks occur in certain waves or periuds, of which the fourth during the present century is at present prevailing in the United States. For several years there have been local epidemics of the disease in widely separated regions, usually in villages and country districts; but in 1896 1897 and 1898 , a slight epidemic occurred in Boston, and in the latter year cases began to be recognised in Baltimore and other towns. From a recent collective investigation, made under the direction of Surgeon-General Wyman, of the United States Marine Hospital Service, ${ }^{1}$ we learn that cerebrospinal fever has prevailed during the past year (as a fule in a mild form) in 27 States, and in the District of Columbia. It is not possible to say that the movement of troops last sum mer had anything to do with the spread of the disease. The limitation for a year or more to one district or city, without spreading to adjacent towns, the localisation even (as at present in Washington and Philadelphia) to special districts of a city, the recrudescence for several seasons in succession, are well-known features illustrated by the present epidemic.

(c) Among specific diseases cerebro-spinal fever stands in some points close to pneumonia. Sporadic cases of both occur at intervals of epidemic prevalence, though in pneumonia they a ce much more numerous, while epidemics of pneumonia, like those of cerebro-spinal fever, are most striking in barracks, asylums, and gaols. Even when not prevailing as an epidemic there may be remarkable house epidemics of cerebrospinal fever. The seasonal relations are the same in both, and the two diseases may prevail together. The abrupt onset, the great frequency of herpes in both, the leucocytosis, "the almost identical characters of the fibrino-purulent exudate in the two diseases" (Netter), the frequent complication of pneumonia in epidemic cerebro-spinal fever and of meningitis in pneumonia are additional points of contact. The degree of contagion is about the same in both diseases, and, lastly, it has been claimed that the organism described in cerebrospinal fever is only a variety or a degenerate form of the pneumococcus.

On the other hand, Leichtenstern, speaking against the view that the pneumococcus.is the cause of epidemic cerebrospinal fever, says:
Pneumonia is a disease spread over the entire earth, and appears at an times, there being no land immune from it. Epidemic meningitis is very rare, and in many countries is still unknown. Croupous pnoumoning age; epidemic meningitis is a disease which affects children and young people; beyond 35 there is slight disposition to it. Croupous pneu; monia has a typical course and a crisis ; epidemic meningitis (Quoted by Councilman, Mallory and Wright. ${ }^{2}$ )

\section{Bacteriology of Cerebro-Spinal Fever.}

Until within the past few years the etiology of cerebrospinal fever has been obscure, and a majority of observers regarded the organism found in the meningeal exudate as the pneumococcus, or a variety of it. More than twelve years ago, however, Weichselbaum described a diplococcus with special cultural peculiarities, which he regarded as the specific organism of the epidemic form of the disease. Little or no attention was paid to his communication until 1895, when his observations were confirmed by Jaeger. So little notice, indeed, had they attracted that neither Ormerod in Allbutt's System (vol. i, published in 1896), nor Latimer in Loomis and Thompson's System, published in 1897, mention Weichselbaum's organism. The work of Heubner, ${ }^{3}$ in Germany, and more particularly the studies of Councilman, Mallory, and Wright, have fully confirmed the observations of Weichselbaum, whose organism, known variously as the meningococcus and the diplococcus intracellularis meningitidis, is now regarded as the specific cause of the disease. As the bacteriology of the subject is very fully discussed in the monograph by Councilman and his associates, and in the just-issued article by Netter in vol. xvi of the Twentiets Century Practice, I shall confine my remarks to an account of our experience in the study of the cases which have been in my wards during the past year. To Dr. Norman $B$. Gwyn, who has charge of the bacteriological work in $\mathrm{my}$ clinical laboratory, and to Dr. N. McL. Harris, bacteriologist in the pathological department of my colleague, Professor Welch, I am under special obligations for their careful study of the cases during life and after death.

The meningococcus in cover-slips made from the exudate has usually a diplococcus form, and lies within the polynuclear leucocytes; hence the definition "intracellularis" Many of the cells may be stuffed with them. They may also occur free. It stains with the ordinary staining reagents, and is decolorised by the Gram method. It grows best on Loeffler's blood serum, on which it forms " round, whitish, shining, viscid.looking colonies with smooth, sharply-defined outlines, which attain a diameter of 1 to $1 \frac{1}{2} \mathrm{~mm}$. in 24 hours" (Councilman). In cultures it is usually short-lived; but Netter states that Germano has found that it resists. desiccation, and has preserved its vitality to the end even of ninety days. He states also that Neisser has shown that the organism is transportable by atmospheric currents, even the most feeble, and that it is very susceptible to aërial convection. It is found in the cerebro-spinal exudate, rarely elsewhere in the body; but it has been isolated from the blood, the pus from the joints, the pneumonic areas in the lungs, and the nasal mucus.

The organism is feebly pathogenic for animals. Rabbits and mice resist subcutaneous inoculation. Heubner, and Councilman and his associates, have produced typical meningitis by inoculating cultures of the meningococcus beneath the spinal membranes of goats.

Our clinical and pathological experience with the organism. is as follows : In 21 cases which I have seen, the lumbar puncture was made in in. In
3 patients seen in consultation the diagnosis seemed so clear that lumbar puncture was not made. In Cases I and II, both mild, the puncture ws 9 made. in one on the seventh day, in the other on the sixth; but no organisn. 9 were found. Cases III and Iv were admitted late in the disease, and it w: 9 not thought necessary to perform it. Of the ren aining $1_{4}$ cases, in 13 the diplococcus intracellularis was present on cover-slips and in cultures. In the remaining case its presence was doubtful on the cover-slips, and the staphylococcus grew in culture. Of the cases which came to necropsy, 5 in number, the bacteriological results, as given by Dr. Harris, are as follows: Necropsy No. I, IO4 ; meningitis, meningococcus ; the pneumoextensive arthritis, in which the diplococcus intracellularis with found in the blood and joint tissues during life, as well as from the found in the blood and joint tissues during life, as well as from the meninges, post mortem the meningococcus was separated in pure culand the bacillus lactis aërogenes; from the blood in the coronary arteries the pneumococcus. The other organs were sterile. In Case $x$ (Necrops No. $i, 247)$, on which laminectomy had. been performed, the sping [2008] 
meninges at the time of his death (about two months later) were perfectly normal. At the operation the staphylococcus pyogenes grew in the culture. In No. $r, 314$, a case in which laminectomy was performed, th meningococcus was isolated from the meninges, both during life and and the staphylococcns pyogenes albus, were also isolated from and the staphylococcns pyogenes albus were also isolated; from a coceus pyogenes aureus, from the spleen the staphylococcus pyogenes citreus, and from a thrombus in the superior longitudinal sinus, and from the pelvis of the left kidney the streptococcus septicus liquefaciens (Sternberg). This case had a diphtheritic and hæmorrhagic cystitis. No. r,364, a very protracted case, in which death occurred in the twelfth week, and from which the meningococcus had been isolated from the spinal fluid twice, from the meninges post mortem the streptococcus pyogenes and the bacillus coli communis were isolated ; and the staphylococcus pyogenes aureus and the bacillus coli communis from the bronchopneumonic areas in the lungs. In a fatal case outside the hospital the in cultures from the cerebral meninges. There was no focus of pneumonia in the lungs.

The following memoranda by Dr. Harris are of interest :

Cultural methods employed. In the first cases we followed the method recommended by Councilman-namely, the use of blood serum "slants." Such were found to be almost useless on account of contaminating organisms, for out of a series of 8 tubes only r gave pure cultures.

1n the othalin 5. per cent. glycerine agar. This procedure gave entire satisfaction in that organisms in the exudate (Cases $1,3^{14}$ and $\mathrm{x}, 3^{24} \mathrm{~A}$ ) were readily sepsrated and classified.

Upon certain points
Councilman's article.

(a) We have never seen as Jaeger states, a capsule formation, nor in ans case unassociated with other pyogenic organism were we able tc stain the specific organism by Gram's method.

(b) The cases coming to necropsy at the height of the disease furnishe upon cover-slip and culture abundant evidence of the presence of th meeble results, or, as in Case $x, 364$, no evidence at all.

(c) The biological features of our several cultures gave practically the same results as Councilman obtained, except that in a few cases. after carrying the organism through several generations, litmus milk was found to be slightly bleached, but witi no evidence of acid formation.

Regarding potato culture, Councilman's statement of an invisible growth on this medium should be expanded by remarking that microscopic pre parations invariably show evidences of moderate incr

At no time has blood serum culture been observed to bear any striking resemblance to a growth of pneumococcus on a similar nedium

As in Councilman's experience, great variations in viability of the organisms was noted. But it is grorthy of mention that where there is condensation water in the tube and growth present in it, the organisms as a rule will be found to be alive many days after life has ceased in the growth upon the surface of the medium.

Staining Reactions. - Supplementary to Councilman's remark upon focal staining points when using methylene blue, it might be said that if old coeffler's methylene blue solution is used, the coloration of that focal point is a decided violet-red.

Animal Experiments. - Subcutaneous inoculation in rabbits and mice (white and grey house mice) was resisted in every case. In rabbits inocuated beneath the dura mater, or into the ventricles of the brain, deatl occurred in all within fourteen days to six weeks, and in no irstance were we able to find evidences of gross pathological lesions, nor the presence greatly emaciated. died in from three to four days, in most cases with general septicamia. Special Remarks.- Regarding Jaeger's observation of the occurrence of Special Remarks.--Regarding Jaeger's observation of the occurrence of
chains of cocci in exudate or spinal fluid, it may be said that it has not been observed, but on blood serum growths it was quite common to find chain formation with the unstained light line of cell division running uncommon amongst the pyogenic staphylococci.

Attempts to isolate the specific organism from any other site than the spinal fluid and the meninges of spinal cord and brain have in every case been attended with failure. This is especially noteworthy in Case $\mathbf{r}, \mathbf{1 8 9}$, which presented arthritis. Alarge quantity of the sero-purulent fluid was inoculated, incubated for three days, and the plates were found sterile, and yet cover-slips of the fluid showed considerable numbers of intracellular diplococci. The heart's blood of this case failed to give positive results at necropsy, although during life the organism had been oroncho-pneumonic foci have always given the pneumococcus, either alone or with the pyogenic staphylococci.

One case, No. $i, 3{ }^{4}$, brings out a possible source of error in the use of Gram's stain upon the cover-slip preparations of the exudate in cases where a secondary infection may be present. The cover-slips from the meningeal exudate in this case were examined by Gram's method before cultures were ready, and cocci were found which did not decolorise, thus causing some surprise, but it was cleared of doubt by finding the staphylococcus pyogen

Case 1,325A is most interesting in that from the exudate on the cerebral meninges the meningococcus, the pneumococcus; and the bacillus coli communis were isolated and positively identified. A mouse was inocucondensation-water growth, and succumbed upon the third day with a

Another mouse received 5 ccm. of a strong suspension in Davham's medium of a twenty-four-hour-old agar-agar culthre of the pneumococcus, when stained by Welch's capsule stain, exhibited well-defined capsules
Microbic Association in Cerebro-Spinal Fever.-Councilman, Mallory, and Wright state that

Mixed infections with other organisms were not uncommcn. The pneumococcus was found seven times, once in connection with Friedcocci were occasionally found

Netter refers to this point in the following terms :

In a very small number of cases observers have reported finding at the same time with the pneumococcus or the diplococcus the stapliylococcus pyogenes, the streptococcus, the colon bacillus, the bacillus proteus, and the capsulated bacillus. These microbic associations are always secondary, however, and we must refrain from regarding any but the two agents

In the report on the bacteriology of the post-mortem examinations by Dr. Harris, the frequency and number of microbic associations will have been noticed. Three points are of interest in this connection: In chronic cases, as $\mathrm{x}$ and XvII of our series, the diplococcus intracellularis may no longer be present ; we have obtained it, however, for the first time on the twenty-fifth, thirty-first, and forty-first day of the disease. Of the two cases referred to, No. xvir presented a most typical picture of the disease, and the diplococcus intracellularis was obtained in the spinal fluid on the thirty-first and thirty-fifth days. At the necropsy the meninges showed a moderate amount of exudate, and there was fluid (purulent) in the posterior horns of the ventricles. The diplococcus intracellularis was not found, but the streptococcus pyogenes and the bacillus coli communis were isolated from the meninges.

Case $x$ is of particular interest, and I give here a brief summary of the history :

A sailor, J. F., aged 25 , was admitted October 29 th, r898. The illness had begun on October 26 th with swelling and pains in the right ankle, and the next day the left ankle became swollen, and then the right hip; he was able, however, to walk to the hospital. On admission there were also redness and tenderness of the left wrist. For the first week we were very doubtful as to the nature of his trouble. The temperature was between ${ }_{10}^{\circ}$ and 10 ${ }^{\circ}$ and we rather suspected typhoid fever. On the $3^{\text {rst he }}$ complained of pain in the back and hips, and on November ist it was noticed that his head was in a very retracted position. On November 4 th move his legs. a thick creamy pus removed, which showed cells and numbers of diplococci, some in cells, but a majority free and in groups. Dr. Cushing performed laminectomy, and drained away a large amount of purulent exudate, and irrigated the spinal meninges with normal salt solution. Cultures made from the fluid obtained by the lumbar puncture and at the operation grew the staphylococcus pyogenes aureus. From the history and symptoms the case was doubtless on

More frequently the pneumococcus has been found in association with the diplococcus intracellularis. In our series it was present only once (Case XIII) in the fluid obtained by lumbar puncture. On the fifth day $20 \mathrm{c.cm}$. of a faintly cloudy fluid were removed, and both organisms grew in culture. On the seventh day the pneumococcus was isolated from the blood. Netter found the pneumococcus associated with the diplococcus intracellularis (post mortem, I take it, though it is not so stated) in 10 of 16 cases of cerebro-spinal meningitis.

A third point is the occasional association of the tubercle bacillus with the diplococcus intracellularis, but so rarely that it is not a point of much practical importance.

On the whole, then, our observations suppcrt those of Weichselbaum, Jaeger, Heubner, Councilman, and others, that in epidemic cerebro-spinal fever there is an organism with special cultural peculiarities which may reasonably be regarded as the exciting cause of the disease. Netter alone, among recent observers, seems to doubt this, and says that he only found the diplococcus intracellularis in 16 out of 39 cases, and in ro of these the pneumococcus was present at the same time. His most recent statement in the Twentieth Century Practice (1899) is worth quoting :

Certain observers, and more particularly Jaeger and Heubner, believe that the diplococcus intracellularis is the sole pathogenic agent in influence whatever in the production of true pneumococcus has no Councilman is a little less emphatic, although he appears disposed to Councilman is a little less emphatic, although he appears disposed to view. The pneumococcus can, without any doubt, cause meningitis, and in spite of Heubner's experience, the rofle of the pneumococcus has been pneumionia complicating meningitis and of meningitis complicating pneumonia, and the coinoidence with meningitis of many other inflammations which may be excited by the action of the pneumococcus, imply

He seems to think that the diplococcus intracellularis may be-a degenerate form of the pneumococeus, and in support 0 his view cites: 
Its intracellular position is evidence of a phagocytosis going on, the impossibility of.ciltivating most of the individuals, as shown by the small number of colonies obtained, its decolorisation by Gram's method, and it usual innocuousness in animals. I would not, however, insist upon this opinion, or lay too much stress upon the arguments just mentioned, for

It does not seem to be altogether clear from certain of Netter's observations that he has been dealing with the meningococcus. Thus in his communication ${ }^{6}$ the coccus which he found in the 8 cases was rounded, usually in pairs, sometimes in short chains; at other tines capsules were seen. They stained by Gram's method, and in bouillon long chains developed, and mice resisted infection when inoculated subcutaneously. He concludes from these observations that the meningococcus is nothing more than a degenerate pneumococcus. Dr. Harris, in a note on this, remarks :

Such conclusions are entirely unwarranted, or, if his observations are correctly stated, he evidently had the veritable pneumococcus to deal with, and by some strange mode of reasoning concludes that he lias the meningococcus in his series of cases, and that

Netter's position is most illogical and confusing. Throughout the article in the Twentieth Century Practice-in many ways one of the very best in literaure-he assumes that the specific disease, cerebro-spinal fever, may be caused either by the pneumococcus or the diplococcus intracellularis. That a primary cerebro-spinal meningitis may be due to the pneumococcus is universally acknowledged; but it is in the highest degree unlikely that a remarkable specific affection like cerebro-spinal fever should be caused by two different organisms. Towards the close of the article the inconsistency of this view seems to impress him, for he says certain peculiarities "prevent us from concluding that the two diseases (that is, pneumonia and cerebro-spinal fever) are absolutely identical."

Diagnosis of Cerebro-Spinal Fever.

Meningitis is not always easy to recognise clinically. Mortifying post-mortem disclosures are even more common with it than with pericarditis. The unexpected meningitis of pneumonia, of Bright's disease, and of other chronic affections, teaches us how latent may be the process; on the other hand, who has not in typhoid fever and in pneumonia made a positive diagnosis of meningitis, and has found post mortem the cerebro-spinal membranes perfectly free? Sooner or later the truth of Stokes's dictum is brought home to each one of us : "There is no single nervous symptom which may not and does not occur independently of any appreciable lesion of the brain, nerves or spinal cord." The anatomical classification of lepto-meningitis is not very satisfactory, and, as I have stated, there is great lack of uniformity in the terms employed. The Nomenclature of the Royal College of Physicians recognises four groups-purulent, tuberculous, syphilitic, and cerebro-spinal fever. An etiological classification, possible to-day in part, is of necessity incomplete. In the annexed diagram I have made a provisional grouping of the chief forms:-

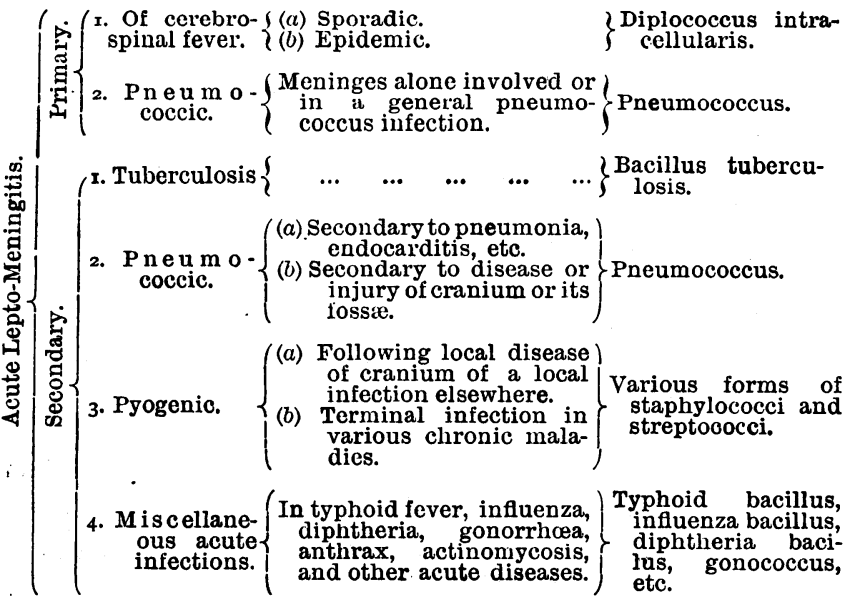

The acute primary lepto-meningitis in a large proportion of all cases follows an infection with the diplococcus intracellularis or the pneumococcus. It will be a great gain if
Still's observations-to which I shall refer later-on the posterior meningitis are confirmed. I do not know where Quincke's meningitis serosa should come in an etiological grouping. Of 3 cases diagnosed as such in our records, in 1 a micrococcus was isolated, but nothing further is stated as to its identification. It is highly improbable that, as Netter holds, cerebro spinal fever, either in the sporadic or epidemic forms, is caused by the pneumococcus. The body of evidence accumulated within the past few years in Germany and America is strongly in favour of the specific relationship of a definite, easily-recognised organism, the diplococcus intracellularis. The pneumococcus may produce a primary meningitis, and pneumonia at certain seasons and in certain regions may frequently be associated with this lesion; but both clinically and bacteriologically this form can be distinguished from the disease under consideration.

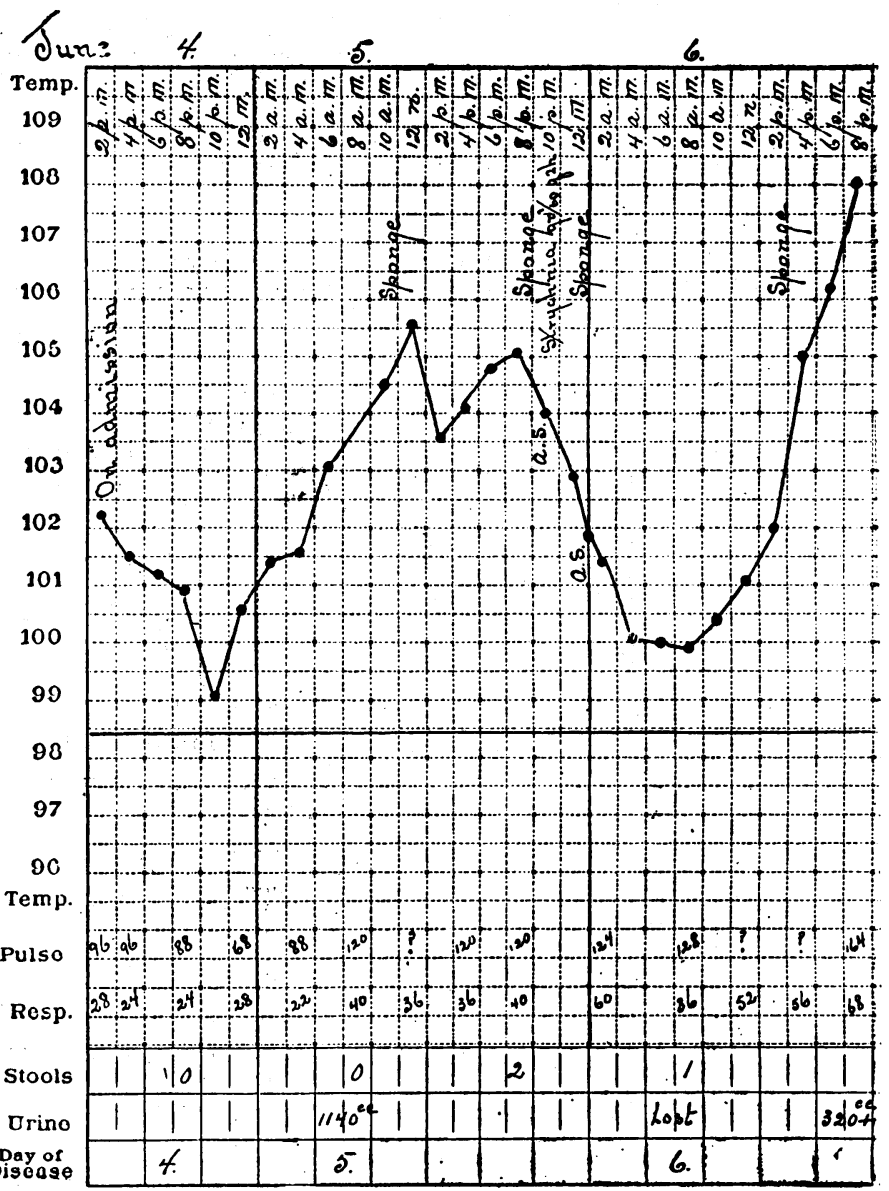

Chart I, Case vir. E. R.

While the possibility of the occurrence must be admitted, I have not recognised a primary meningitis caused by the pyogenic organisms ${ }^{6}$ or by the tubercle bacillus. The terminal meningitidis I have placed among the secondary forms; and as in the terminal pleurisies and peritonitides, a variety of organisms are found. Even in cerebro-spinal fever the. streptococci and staphylococci, always on the watch for a locus: minoris resistentice, may drive out the original foe, as in. Cases $x$ and $X V I I$ on our list.

To the secondary forms of meningitis in the table I shall not refer, except to call attention to the importance of the pneumococcus in this group also; nor can I discuss within the limits of this lecture the clinical features of the varieties of meningitis, and must confine my remarks to a few specia points in the diagnosis of cerebro-spinal fever, and to a consideration of certain sporadic forms of meningitis.

I. Certain Features in the Symptomatology of Cerebro-Spinal 


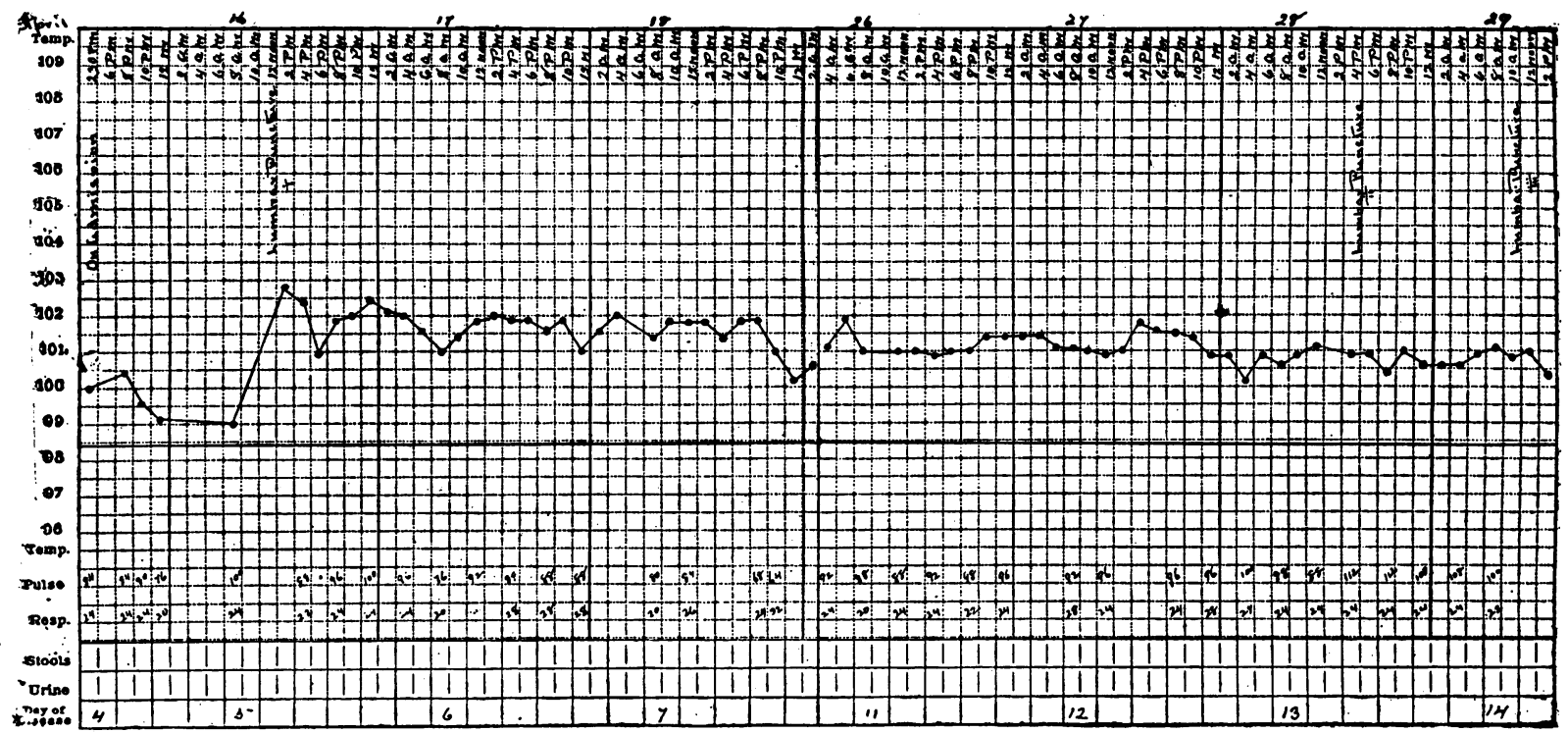

Chart 2, Case Xvito-P. C.

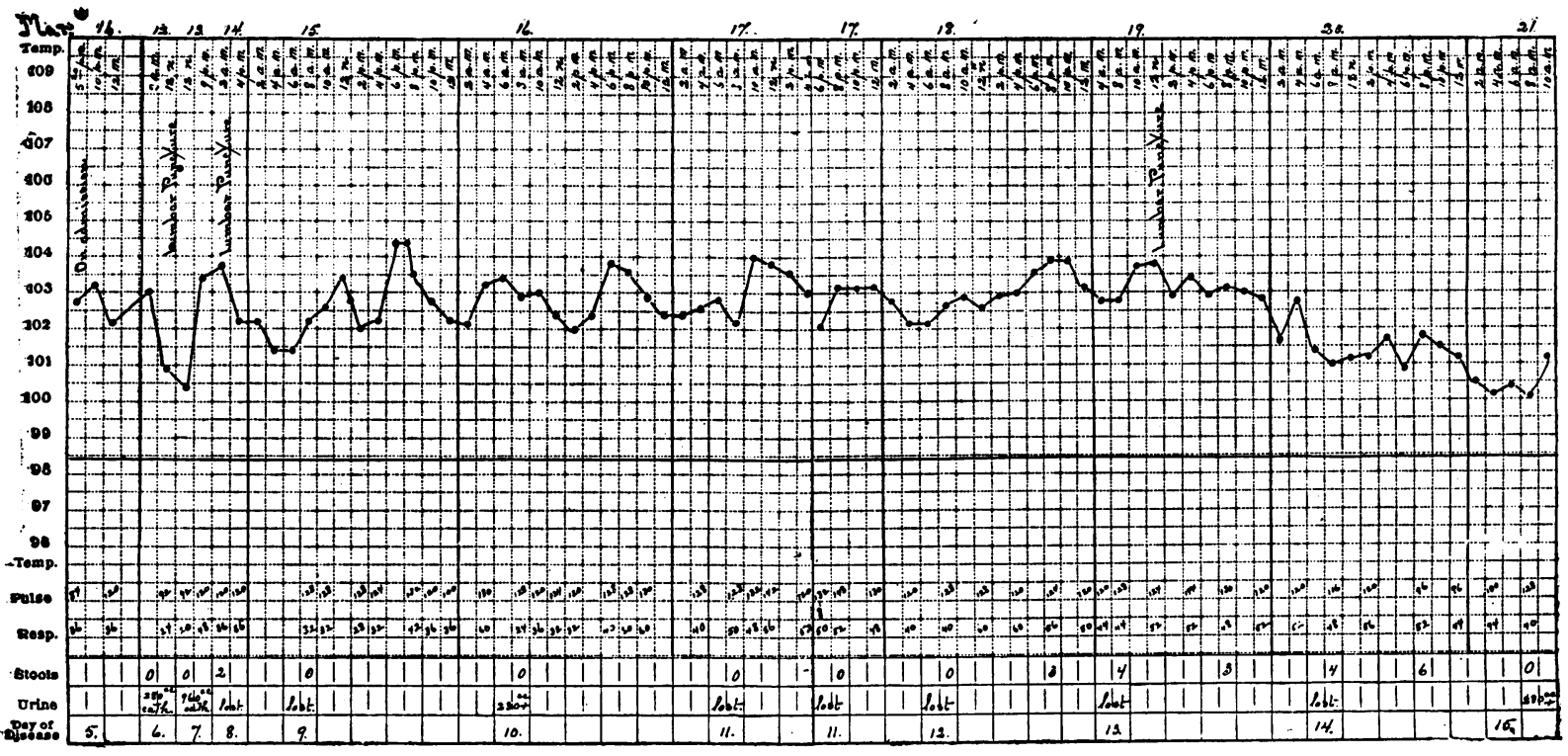

Chart ${ }_{3}$, Case xirr.-O. S.

Fever.-No other form of meningitis presents a symptomatology of the same fulness-general, cerebral, spinal, and peripheral features are present in almost every case. This volvement, in part from the very frequent implication of the nerves, and to some degree from the very chronic course. I shall discuss only a few of the important symptoms.

(a) Onset.- In striking contrast to other forms, particularly the tuberculous, cerebro-spinal fever sets in abruptly. Without warning, while at work, or awakening the patient from a sound sleep, comes the pain in the head, etc. This peculiarity may also be met with in primary pneumococcic meningitis; but in the form secondary to pneumonia, and in that which arises in endocarditis, latency of onset is the rule.

(b) Fever.-There is no constant type of fever in any form of meningitis. In cerebro-spinal fever the pyrexia is very variable, and I have given here a few charts in.illustration. There may be no fever at onset-as shown in Charts 8 and 9 of the sporadic cases. Of our epidemic cases, all had fever on admission.
Irregularity in the fever may be present from the start. There is not a steady rise at first to a fastigium, but even on the third or fourth day the temperature may fall to normal. Chart I shows this extreme irregularity in a two-hour record. The remissions were very pronounced, and the temperature rose to $108^{\circ}$ just before death.

In other cases the course is singularly like that of typhoid fever, and for days the two-hour temperature chart may not show a variation of more than a degree. This is well illustrated in Chart 2 from Case Xvir. This patient (as shown on the lantern slide) had the facial aspect of a case of typhoid fever. Chart 3 from Case XIII shows also a very steady fever with but slight remissions to the time of death on the fifteenth day. Chart 4 from Case $\mathrm{xv}$ presents a still more marked resemblance to a typhoid fever curve, particularly in the daily remissions, giving the spiked chart with which we are all so familiar in the third week of the disease. Intermissions, exacerbations, and great irregularities similar to those in tuberculosis, are very pronounced in the more chronic cases. Chart 5 from Case' Xvi gives the $m$ m 1 ximum 
and minimum daily temperature from the twenty-ninth to the seventy-ninth day. It will be noticed how variable is the time of the highest point of daily fever. On thirteen days the maximum temperature was in the morning - an inverse type, to which many writers have referred, in this disease. The exacerbations are better shown in the two-hour record. In Chart 7 from Case XVI the intermittent type is shown, but the paroxysms are readily distinguished from those of tertian malarial intermittent fever by their prolonged course.

In cases of this sort, when chills occur, the picture is very suggestive of malaria, and one can understand why some of the older writers regarded the disease as a manifestation of paludism. The chart of Case vIII is worth a rassing comment. After a very steady temperature on the ninth, tenth, and eleventh days the record on the twelfth day was normal. On the thirteenth day there was a paroxysm without a chill, lasting from 10 P.M. On June 19th to 4 A.M. on June zist. On the fourteenth and fifteenth days the temperature was normal. On the evening of the latter the fever rose, and the paroxysm lasted through the sixteenth day, and at 4 P.M. the patient complained of decided chilliness. On the seventeenth day no fever. At io A.M. on the eighteenth day the patient had a rigor, in which the temperature rose to $104.5^{\circ}$; the paroxysm lasted more than thirty hours. Then, on the

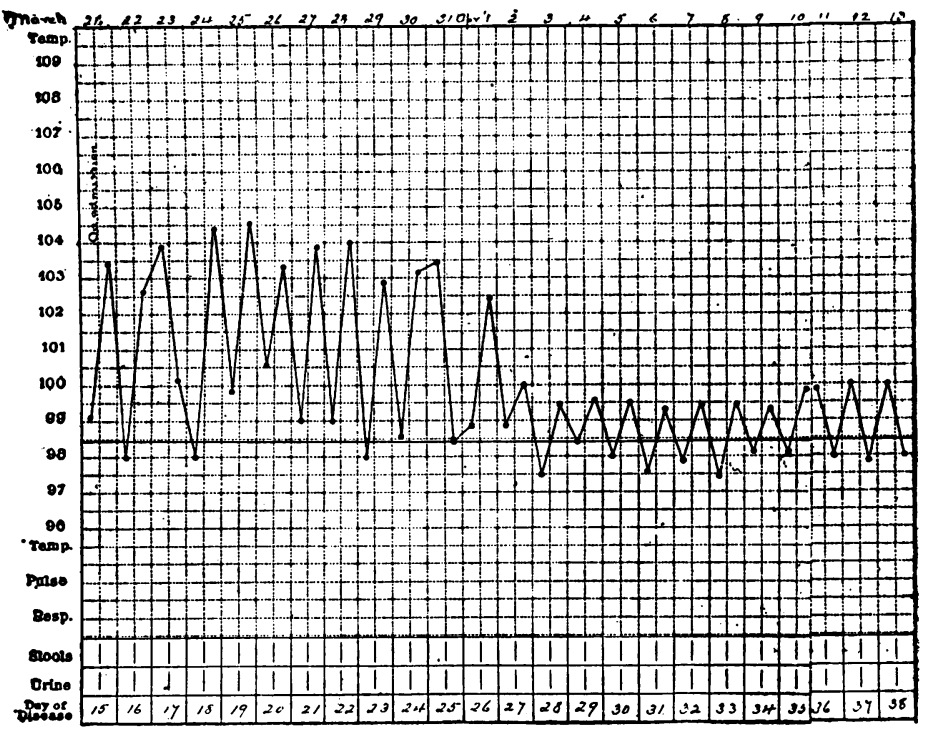

Chart 4, Case xv.-J. N. M.

twentieth, twenty-first, and twenty-second days he had recurring attacks, two chills, the one on the twenty-second, without chill, extended into the twenty-third day. After this the patient's temperature remained normal, and he recovered rapidly. During these attacks he complained a great deal of pain in the head and back. The spleen was not enlarged. The blood was examined daily for the malarial parasites, but none were found. And, lastly, Chart 7 , from Case $x v$, illustrates the relation of the temperature to the lumbar puncture. After the sixth, seventh, and eighth punctures the fever dropped ; after the twelfth it rose.

(c) Skin Rashes.-Various skin rashes are common in cerebro-spinal fever, and form an important feature in the diagnosis. Of 21 cases which I saw during the epidemic, in I3 a skin eruption of some form or other was present. Herpes, of course the most common, was present in 8 cases. A diffuse ery thema about the chest and abdomen, and over the joints, was present in 4 cases. Petechiæ were noticed in 8 cases, extensive in only 3. In 3 cases a very remarkable and peculiar rash was present in the neighbourhood of the joints, particularly over the extensor surfaces of the knees and elbows, and about the ankles. There was a diffuse, livid erythema of great intensity, on which a purpuric herpes developed-a vesicular rash, the individual vesicles of which became filled with blood. As the erythema faded and the vesicles dried, they could be felt as little nodular hemispherical bodies, which persisted for a week or ten days.

(d) Blood.- A careful study of the blood was made in all of our cases. A leucocytosis was present in every instance. In 4 the first blood count was made on the third day, and the leucorytosis was $25,900,14,500,40,800$, and 32,000 per c. cm. The first blood count was made on the fourth day in 4 cases, and the leucocytosis was $26,240,31,800,19,300$, and 7,600 per c. cm. In only 4 cases did the leucocytes exceed 40,000 per c. $\mathrm{cm}$., and the maximum count was on the ninth day in a fatal case 47,000 per c. cm. The leucocytosis persists even in the most protracted cases. In a lad who died in the twelfth week of the disease the leucocytes were 23,000 per c. cm. shortly before death. As a rule there was no special reduction in the red blood corpuscles. In 1 case a moderate anæmia developed, and in the fifth week the red corpuscles were only $3,100,000$ per c. $\mathrm{cm}$. In 1 case the diplococcus intracellularis was isolated from the blood during life.

Leucocytosis has no special value in the differential diagnosis of the various forms of meningitis. I had an impression that it was not so constant in the tuberculous variety $;$ but Dr. Parfitt analysed the records in 11 of our cases, and found that in only 3 was a leucocytosis absent. The lowest count was 6,500 per c. cm. Of 8 cases with leucocytosis, there were 4 with 20,000 or more white corpuscles per c. $\mathrm{cm}$. The highest count was in a child aged $8-24,333$ per $\mathrm{c}$. $\mathrm{cm}$. Altogether the leucocytosis seems not to be so high or so persistent in the tuberculous meningitis.

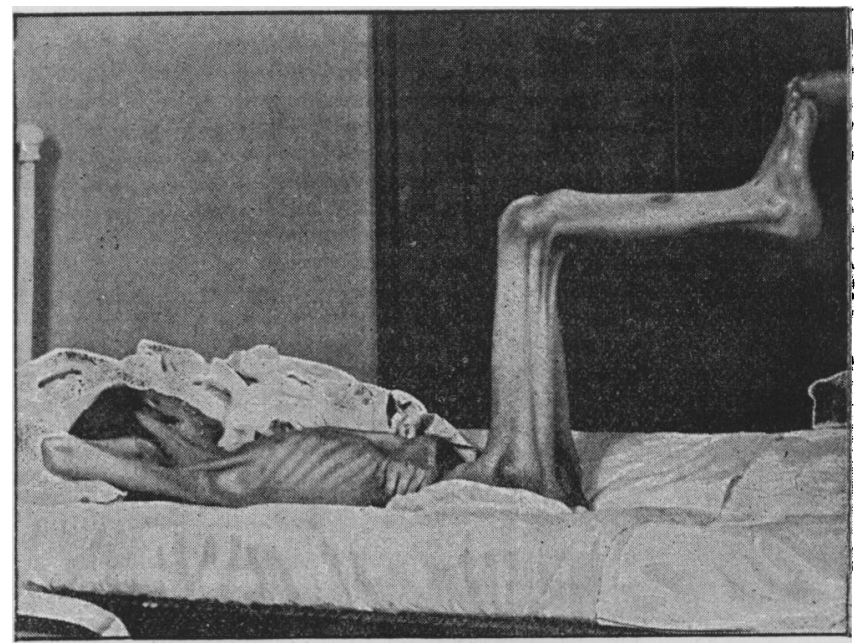

Fig. r.-Kernig's sign, showing the strong contraction of the flexors on attempting to extend the leg.

(e) Arthritis, or more often periarthritis-much more common in cerebro-spinal fever than in other forms-was present in 2 of our cases. In Case vil the joint lesion came on with great rapidity, and by the fourth day he had a multiple suppurative arthritis, resembling an acute pyæmia. The diplococcus intracellularis was isolated from the pus in the joints. In the other (Case $x$ ) the disease began with arthritis.

II. Kernig's Sign.-Described by a Russian physician, and studied in Germany and France, this interesting sign has not attracted the special attention of English and American physicians, though J. B. Herrick, of Chicago, at the last meeting of the Association of American Physicians, spoke of its value. It has been present in all our cases in which it. has been looked for. It is, I think, an old observation that the subjects of protracted meningitis, particularly children, very often lie with the thighs flexed upon the abdomen, and with the legs in a state of partial contracture, so that they are with difficulty extended. To test for Kernig's sign the patient should be propped up in hed in the sitting position, then, on attempting to extend the leg on the thigh there is contraction of the flexors which prevents the full straightening of the leg. On the other hand, in the recumbent posture the leg can be iully extended. Many patients with meningitis are not in a 


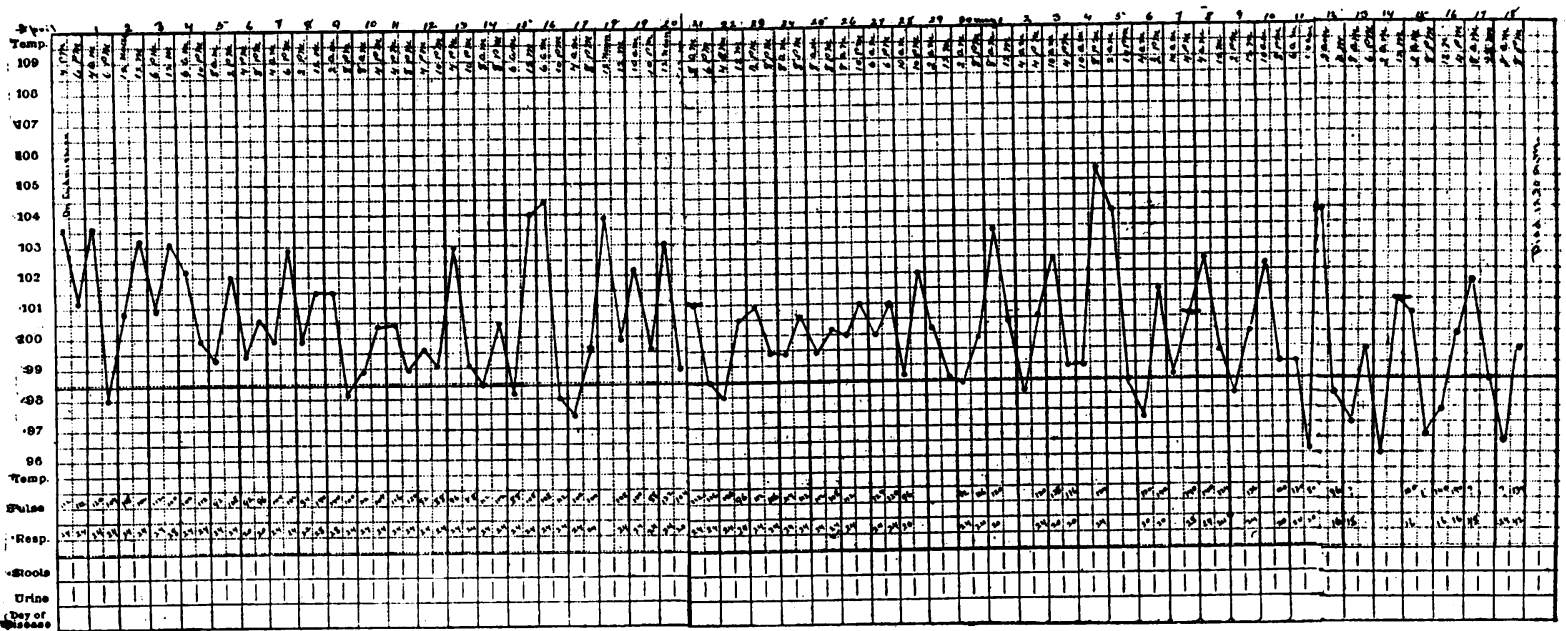

Chart 5, Case xvi.- C. A, K.

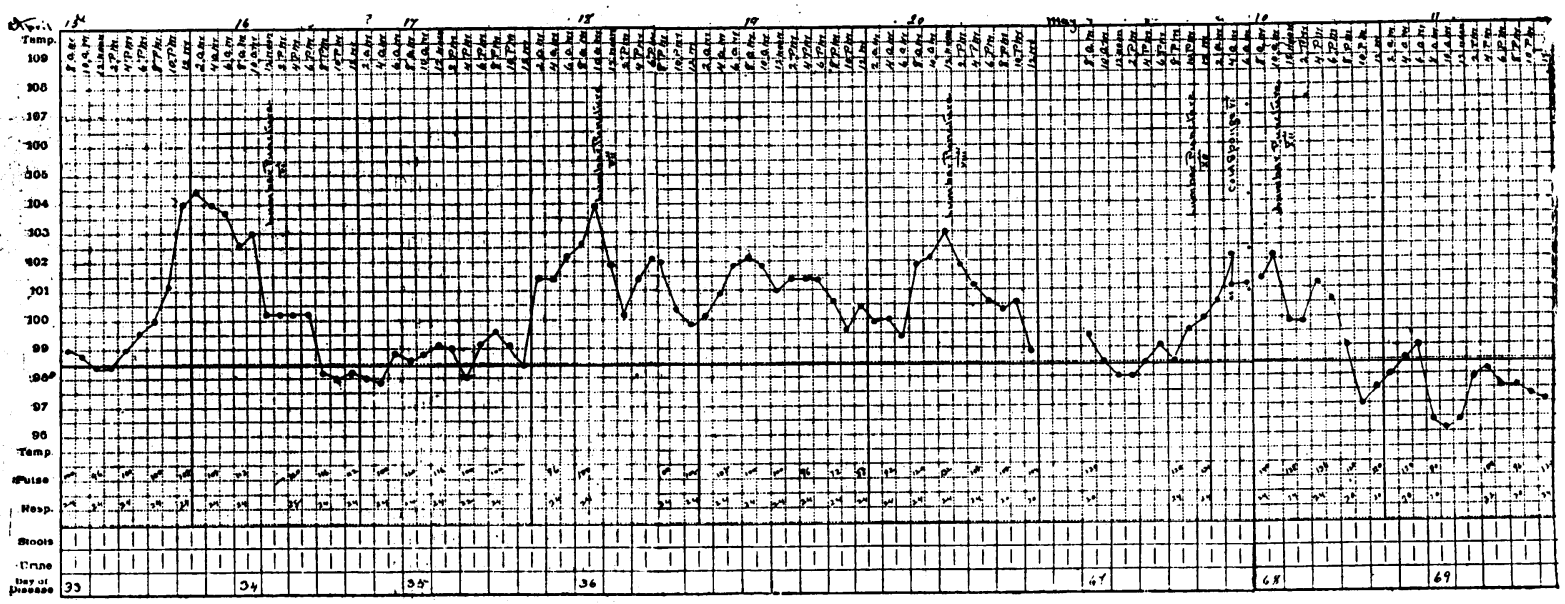

Chart 6, Case Xr.-J. N. M.

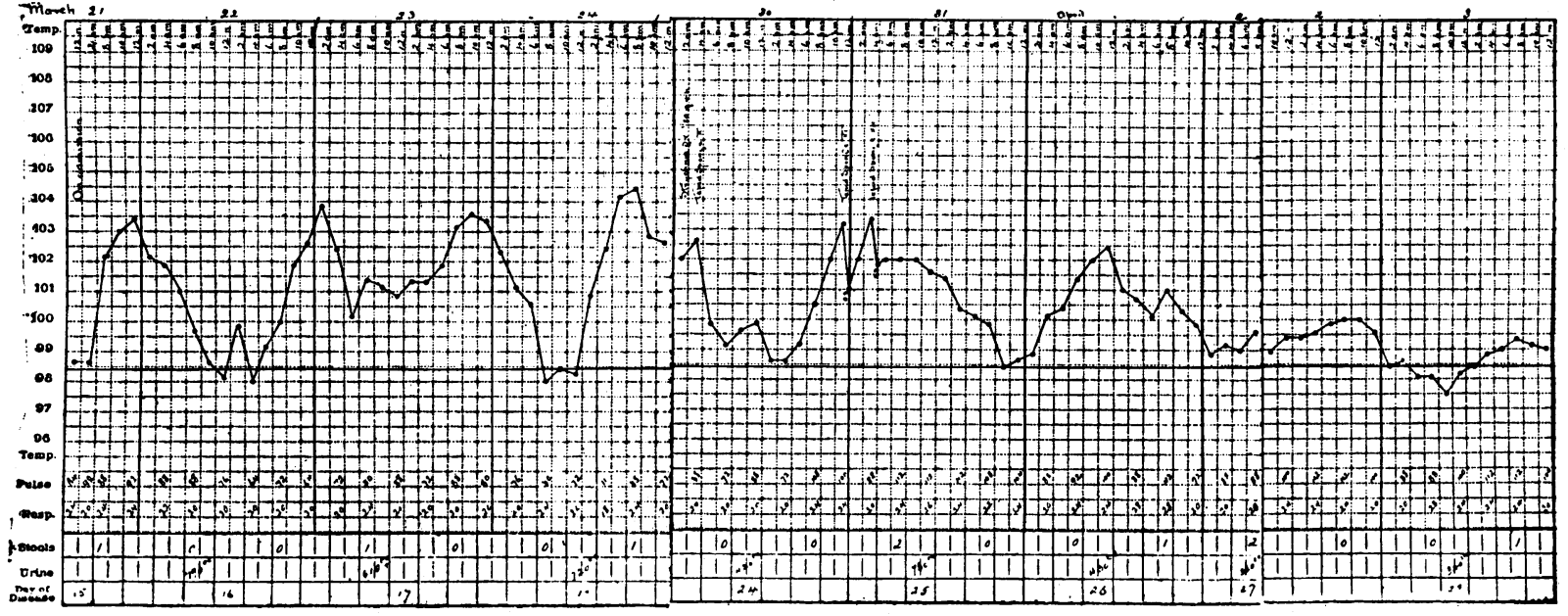

Chart 7, Case xvi. C. A. K.

condition to sit up, and the test can be equally well made by Hexing the thigh on the abdomen, when cn attempting to rxtend the leg, if meningitis be present, the limb cannot be f ully extended, as shown in Fig. I (p. 1521). Friis found the,

sign in 53 of 60 cases, and Netter in 45 of 50 . It is stated to be present in all forms of meningitis when the spinal meninges are involved. The presence of the sign is no indication of the intensity of the spinal 
involvement, as it existed in a very marked degree in a recent case of pneumococcic meningitis, in which there was no positive exudate on the spinal meninges, only a turbid fluid. Netter's explanation of the phenomenon is as follows : "In consequence of the inflammation of the meninges the roots of the nerves become irritable, and the flexion of the thighs upon the pelvis when the patient is in the sitting posture elongates and consequently stretches the lumbar and sacral roots, and thus increases their irritability. The attempt to extend the knee is insufficient to provoke a reflex contraction of the flexors while the patient lies on his back with the thighs extended upon the pelvis, but it does so when he assumes a sitting posture."

III. Lumbar Puncture.-During the past ten years no single measure of greater value in diagnosis has been introduced than Quincke's lumbar puncture. We are now able in a large number of cases to make a prompt decision as to the existence of meningitis, and are further enabled to recognise the form of the disease. I shall not detain you with details of the technique, available now in all textbooks, and recently considered at great length in an elaborate "Referat" by Neurath in the Centralblatt. f. de Grenzgebiete der Medizin und Chirurgie, Bd. I. It is a simple, quite harmless procedure, and in a majority of the cases can be done without general anæsthesia, or with the aid of a local freezing mixture. A dry tap is rare in cerebro-spinal fever; the needle may be plugged with fibrin, or a nerve root may come directly against the orifice. Puncture in the third or fou:th interspace may be negative, while in the second a free flow is secured. In one of our early cases in which no fluid was obtained, the necropsy showed an exudate as thick as butter, with little or no fluid. A first puncture in a case is very often negative. Though simple the technique is, like other procedures of the kind, bettered by practice. Very often at first a few drops of blood flow, then a clear or turbid fluid, either drop by drop, or sometimes in quite a strong stream.

The fluid may be clear, turbid, purulent, or more rarely a brownish yellow or quite bloody. In a great majority of all cases, when meningitis is present the fluid is turbid. In rare instances clear fluid may be obtained when meningitis exists, and in a protracted case the fluid may be turbid at one puncture and clear at the next. Several observers have noted that the fluid may become clear in the intermissions of the disease. A clear fluid may be obtained from a puncture in the second lumbar interspace, while lower down a turbid fluid may be withdrawn. I saw this possibility very well illustrated in a recent post-mortem examination; the fluid in the lower dorsal and upper lumbar regions was perfectly clear, while that in the lower lumbar and the sacral regions of the canal was very turbid and contained numerous flocculent masses.

The fluid should be allowed to flow into a sterilised test tube. When the fluid is at all turbid there is usually a slight sediment and coagulation of fibrin. Cover-glass preparations are made either directly from the turbid fluid, or if the turbidity is slight and the cell elements few in number, after it has been centrifugalised. Cultures should be prepared at the time of making the lumbar puncture by allowing one or two cubic centimetres of the fluid to flow on to a Loeffler blood serum medium.

The amount of fluid obtained varies from a few drops to 130 c.cm. The samples in these flasks show the large amount which may be removed, one 126 c.cm., another II 2 c.cm.

Wentworth claims that within certain limits there is some relation between the degree of turbidity of the fluid and the severity of the symptoms, and this was borne out by our more limited experience. Even in the most protracted cases the turbidity may persist. Only once was a clear fluid removed in an exacerbation. On several occasions a bloody fluid was removed in Case Xvill. In the wards at the same time was a remarkable instance of septic meningitis in which, aiter turbid fluid had been withdrawn at one puncture, at a subsequent one, before death, blood flowed from the needle, and post mortem there was extensive meningitis and a rupture of the basilar artery with hæmorrhachis.

The number of the organisms found bears no constant relation to the intensity of the symptoms. In acute cases they are present, as a rule, in large numbers. The later the disease, the less likelihood is there to find the diplococcus intracellularis in the fluid; but we have found it in the second, third, fourth, filth, and seventh week. The following are some of the days on which the organisms were found: seventeenth day, fourteenth day, twenty-fifth day, thirty-first day, forty.first day, and nineteenth day.

Has the lumbar puncture any therapeutic value? Williams, of Boston, states that he has seen beneficial effects, and there are a few cases in the literature in which the severity of the symptoms were promptly mitigated by the removal of $a$ variable amount of the spinal fluid. Wentworth (whose experience has been very large), speaking of these cases, says: "I have never seen any such cases, though constantly on the watch for them. A temporary relief, lasting for a few hours, has followed the operation in a few cases, but the same remissions frequently occur without any treatment." Netter says that he has seen convulsions, which had lasted for a long time without intermission, cease alter the withdrawal of only. about 2 drachms of fluid.

We have given this point our closest attention, and many times have performed the puncture directly for its supposed benefit. In Case VIII the note reads: "Much better after the first puncture ; brighter every way." Case IX, puncture at II A.M.: "Patient's condition has greatly improved; the muttering has ceased, and the irregular movements are less marked." To Case xvi I may refer more fully. It was an example of the chronic form in which the patient lingered for nearly three months. The accompanying table has been prepared by Dr. Marshall, one of my house-physicians. It gives the day of the disease, the amount of fluid withdrawn, the character of the exudate, the microscopical appearances, the culture results, the leucocytosis, and the effect (?) of the puncture on the temperature. The first puncture, made on the twenty-ninth day of the illness, was negative. From the second puncture 100 c.cm. of a yellowish turbid fluid were withdrawn, which contained pus cells and intracellular cocci

Kratz-Lumbar Puncture Chart.-Case xvi.

\begin{tabular}{|c|c|c|c|c|c|c|c|}
\hline $\begin{array}{l}\text { Puncture } \\
\text { No. }\end{array}$ & $\begin{array}{c}\text { Day of } \\
\text { Disease. }\end{array}$ & Amount. & Character. & Microscopically. & Cultures. & Leucocytosis. & Temperature. \\
\hline $\begin{array}{r}1 \\
2 \\
3 \\
4 \\
5 \\
6 \\
7 \\
8 \\
9 \\
10 \\
11 \\
12 \\
13 \\
14 \\
15 \\
16 \\
17 \\
\end{array}$ & $\begin{array}{l}29 \\
31 \\
35 \\
37 \\
40 \\
44 \\
46 \\
48 \\
54 \\
59 \\
59 \\
62 \\
68 \\
70 \\
73 \\
75 \\
75\end{array}$ & $\begin{array}{c}\text { Negative } \\
\text { 100 c.cm. } \\
125 \\
90 \\
85 \\
126 \\
100 \\
50 \\
30 \\
\text { Negative. } \\
\text { 100 c.cm. } \\
60 \\
3 \\
75 \\
\text { Negative. } \\
\text { Negative. } \\
8 \text { c.cm. }\end{array}$ & $\begin{array}{l}\text { Yellow ; turbid. } \\
\text { Yellow ; turbid. } \\
\text { Pale yellow ; turbid. } \\
\text { Pale yellow. cloudy. } \\
\text { Pale yellow ; cloudy. } \\
\text { Pale yellow t turbid. } \\
\text { Pale yellow ; turbid. } \\
\text { Pale yellow ; turbid. } \\
\text { Cloudy. } \\
\text { Cloudy; blood-tinged. } \\
\text { Cloudy. } \\
\text { Cloudy. }\end{array}$ & $\begin{array}{l}\text { Pus cells; intracellular diplococci. } \\
\text { Pus cells; no cocci. } \\
\text { Pus cells; no cncci. } \\
\text { Intracellular; diplococci. } \\
\text { Pus cells; no cocci. } \\
\text { Pus cells; no cocci. } \\
\text { Pus cells; no cocci. } \\
\text { Pus cells; no cocci. } \\
\text { Pus cells; no cocci. } \\
\text { Pus cells; no cocci. } \\
\text { Pus cells ; no cocci. }\end{array}$ & 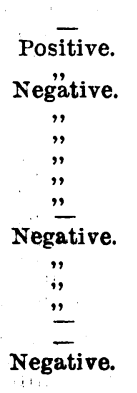 & $\begin{array}{l}- \\
17,400 \\
25,300 \\
19,900 \\
28,000 \\
15,000 \\
22,000 \\
19,000 \\
23,600 \\
= \\
18,000 \\
32,000 \\
47,500 \\
23,000 \\
=\end{array}$ & 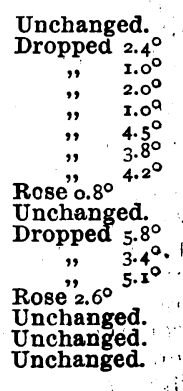 \\
\hline
\end{tabular}


in abundance, and the cultures were positive. In all seventeen punctures were made between the twenty-ninth and the seventy-fifth day of the disease, of which fourteen were positive. A turbid pale yellow fluid was removed at each tapping. On five occasions $100 \mathrm{c.cm}$. or more were obtained, once 125 c.cm., and once $126 \mathrm{c.cm}$. Following the two first effective tappings the patient appeared better, the temperature dropped, and he seemed much brighter, but he soon became worse, and the fever rose. Following the sixth, seventh, eighth, and eleventh punctures the temperature fell $4.5^{\circ}, 3.8^{\circ}, 4.2^{\circ}$, and $5.8^{\circ}$. There was no change in the general condition, though he sometimes was a little brighter, and the drop in fever followed so directly that it seemed ouly natural to attribute the good results to the lumbar puncture. The thirteenth punc. ture was practically negative, yet the temperature fell $5.1^{\circ}$, and after the fourteenth tapping the temperature rose $2.6^{\circ}$. 'Evidently not the withdrawal of the fluid, but the peculiar character of the disease, already spoken of, was responsible for the remissions. The chart illustrates further the persistence of the leucocytosis, and the sterile characters of the fluid after the forty-fourth day. I have here for demonstration the lumbar portion of the cord and its membranes. The position of the last puncture can be seen, and the repeated operations have caused a slight hæmorrhagic pachymeningitis.

\section{Sporadic Cerebro-Spinal Fever.}

To what extent do isolated cases of cerebro-spinal fever occur in the intervals between the epidemic prevalence? What is the nature of the primary suppurative meningitis which is met with from time to time in all communities? Neither hospital statistics nor the ordinary death returns give trustworthy answers to these-questions. The diagnosis of meningitis is often doubtful, and it is very probable that a great many cases are returned as cerebro-spinal meningitis which are the cerebral forms of typhoid fever or of pneumonia. It is surprising how much confusion there is in the diagnosis of the different forms, and the urgent need of a more accurate recognition of the varieties is well shown by the number of terms used in the tabulation of cases. Thus, not to speak of the taberculous form, which is often spoken of as basilar meningitis, the terms most frequently used are acute meningitis, meningitis simplex, purulent meningitis, and septic meningitis. In a recently-issued report on sporadic cerebro-spinal meningitis from the Philadelphia Hospital, by L. N. Boston, ${ }^{8}$ exclusive of the tuberculous variety, the following names occur as designating the varieties of acute meningitis: lepto-meningitis, basilar meningitis, pachymeningitis and basilar meningitis, basilar and lepto-meningitis, hæmorrhagic meningitis, and purulent meningitis.

To reach uniformity in this matter is not at present possible, but we may welcome the steps which have been taken towards separating the different forms of the disease.

In the last Census Report of the United States the returns for the census year 1890, a year in which cerebro-spinal fever so far as I know, did not prevail to any extent in epidemic form, the number of deaths reported from cerebro-spinal meningitis were 3,333 , a large percentage of these during the first year of life.

From the Fifty-Ninth Annual Report of the RegistrarGeneral, 1896, I gather that the deaths from cerebro-spinal fever in England from 1877 to 1896 (inclusive) have only once exceeded 50 per annum. There has been a great reduction in the return since 1887,233 cases for the ten years ending 1896 , against $\mathbf{4 0 6}$ for the previous decade. In scotland there were only 6 deaths from cerebro-spinal fever in 1895 , and 5 in 1896 In Ireland there. were 76 deaths from this cause in 1896, and the same number in 1897 .

From the records of two large metropolitan hospitals I have gleaned the following figures: At St. Bartholomew's from 1860 , cases with the diagnosis of cerebro-spinal meningitis occur in the years $1872,1888,1889,1890,1892,1893,1895$ $1896,1897-21$ in all. In many years the word meningitis alone is used, in others the word spinal meningitis and meningitis simplex. Ormerod ${ }^{9}$ gives an exceedingly interesting report on 10 fatal cases from this hospital ; 4 of them occurred between March and June. 1890, at which time epidemic meningitis prevailed in the Eastern Counties. At St Thomas's since 1873 cases with the diagnosis of cerebro-spinal meningitis occur in the years 1895 and $1896 \rightarrow 7$ cases in all. In the wards of the Royal Infirmary, Edinburgh, there were in 1891 3 cases diagnosed as cerebro-spinal meningitis, in 1892 2 cases, in 1893 I case.

In the United States and Canada the occurrence of sporadic cases of cerebro-spinal fever in the intervals between epidemics has long been recognised. In Montreal I performed post-mortem examinations on at least 3 cases of acute purulent cerebro-spinal meningitis not associated with pneumonia or endocarditis, and not following otitis media or injury. As illustrating the liability to error, I may mention a case in which the diagnosis of cerebro-spinal meningitis was made in malignant small-pox; in another instance, with the same diagnosis, the case was a very acute typhoid fever, fatal within a week. In Philadelphia I saw only 3 cases.

At the Boston City Hospital, from 1880 to 1896 , there were 39 cases diagnosed as cerebro-spinal meningitis, with a mortality of 59 per cent. It is interesting to note that during the first five months of 1897 , when the epidemic began, there were 42 admissions - a larger number than in the previous seventeen years. That the disease lingers in a city indefinitely after an outbreak has been the common opinion of all students of the disease, and anyone who has had a large post-mortem experience has met with sporadic instances of extensive suppurative meningitis which he has not been able to regard as secondary to any existing condition. The Philadelphia records are interesting in this respect. As collected by Stille from 1863 the figures illustrate very well the periods in which there were slight outbreaks-namely, 1863.64 and $1872-73$. Pepper ${ }^{10}$ completed the figures to 1892 and Dr. Abbott has sent me the figures up to date. They show from 1884 a progressive decline in the number of cases, which may in part have been due to more careful diagnosis. From this year, when there were 124 deaths, there was a gradual decline, and in 1891 there were only 23 deaths. From that year the figures as sent me by Dr. Abbott are as follows: 1892, 22 cases ; 1893, 35 (ases ; 1894, 18 cases ; 1895, 17 cases ; 1896, 7 cases; 1897, Io cases; 1898, 24 cases; 1899 (to and including April 3 oth), 89 cases. Here again it is interesting to see how quickly the epidemic prevalence of the disease is manifest. During 1898 the disease was not recognised as prevalent in Philadelphia. During the present year a number of cases have occurred, and the deaths during the first four months have been 89 .

In Baltimore the figures for the past six years are as follows: 1893,59 cases ; 1894,26 cases; 1895,27 cases; 1896 32 cases; 1897,22 cases; 1898,70 cases; during this year the prevalence of the disease was recognised as occurring in a mild form through the city. During the first four months of the present year there has been an increase, and the deaths for this period have been 27

Sporaaic Cerebro-Spinal Fever at the Johns Hopkins Hospital.In the spring of 1898 the first cases of the epidemic form were admitted to the hospital, and in all eighteen cases rave been under treatment. Prior to this date only four cases had been admitted which we regarded as sporadic forms of cerebrospinal fever, three within a few days of each other in 1893 and one, a chronic case, in 1892 , which has been reported."

CASE I.-P. R. (Hospital No. 7253), aged 57, admitted May ist, 1893. The patient had been drinking heavily, and when admitted was delirious mumbling and muttering to himself, and picking at the bed-clothes, and he resisted actively any attempt to examine him. His friends stated that he had been in this condition for about a week. His temperature on admission was $97.5^{\circ}$, pulse 68, respirations 16 . He had no skin rash and no delirium tremens. As shown in Chart 8 on $p$. 1525, he had no delirium tremens. As shown in Chart 8 on p. 1525, he had no fise to ro $5^{\circ}$; the respirations increased from 28 to 40 ; the pulse rose to $\mathrm{ni2}$. rhroughout the 6 th the temperature remained high, falling on the cyanosed, could not be be roused, and he died at $8 \mathrm{~A} \mathrm{M}$. on the 7 th.

The necropsy (No be bhowed extensive yellowish purulent exudate over The necropsy (No. 413) showed extensive yelowish purasent and along the the hemispleres. There was a turbid exudate at the base and along the cord was not examined. Cover-slips showed rounded cocei in pairs, cord was not examined. Cover-slips showed cells. Cultures showed diplobacilli. The lungs showed slight bronchopneumonia, and the plpouacili. The lungs sho

CASE II. -W. F., aged 27 (Hospital No. 7282), admitted May 3rd, 1893. The patient was ap parently very deaf, and was noisy and delirious when admitted and he had no friends from whom any history could be dotained, The temperature on admission was $99^{\circ}$ pulse 86 , respiraations 20 . During the night he became very noisy and delirious, and the pulse 2o. During the night he following morniug examination of the thoracic and abdominal organs showed nothing of any special moment. 
The spleen was not palpable. The temperature was $99^{\circ}$, pulse $96^{6}$ respirations 24. On the evening of the 4 th and throughout the night the patient became very noisy and had to be transferred to the isolating ward. It was noticed on admission that he was very deaf, and this to rise, and at $6 \mathrm{P} . \mathrm{M}$. reached $102,5^{\circ}$. The pupils were equal the pulse was 96 , irregular. The patient resisted strongly any attempt to bend the head, and the neck was held very rigid. There was general hyperresthesia, and he cried out whenever he was touched. A petechial rash was noticed that afternoon for the first time over the abdomen. At 8 P.M. the temperature rose to $103^{\circ}$ (Chart 9 '. He became very much worse; the pulse became more rapid, $11_{2}$, very irregular. and he died a little after 2 in the morning.

Necropsy (No. 4r2). A purulent greyish yellow exudate over the hemispheres, also along the pons and medulla, and the whole length of the spinal cord, which in the lumbar region reached a thickness of $8 \mathrm{~mm}$. The ventricles were distended with a turbid vellowish fluid. In the posterior cornua there was a greenish exudate. Cover-slips from the meninges showed numerous leucocytes and large cells containing round diplococci. Pus from the ventricles showed many cocci, single and in pairs, chiefly in cells. Some of the larger cells were packed with these cocci. Cultures were negative Inoculations of $a$ rabbit and a mouse were also negative. At that time we had not recognised the

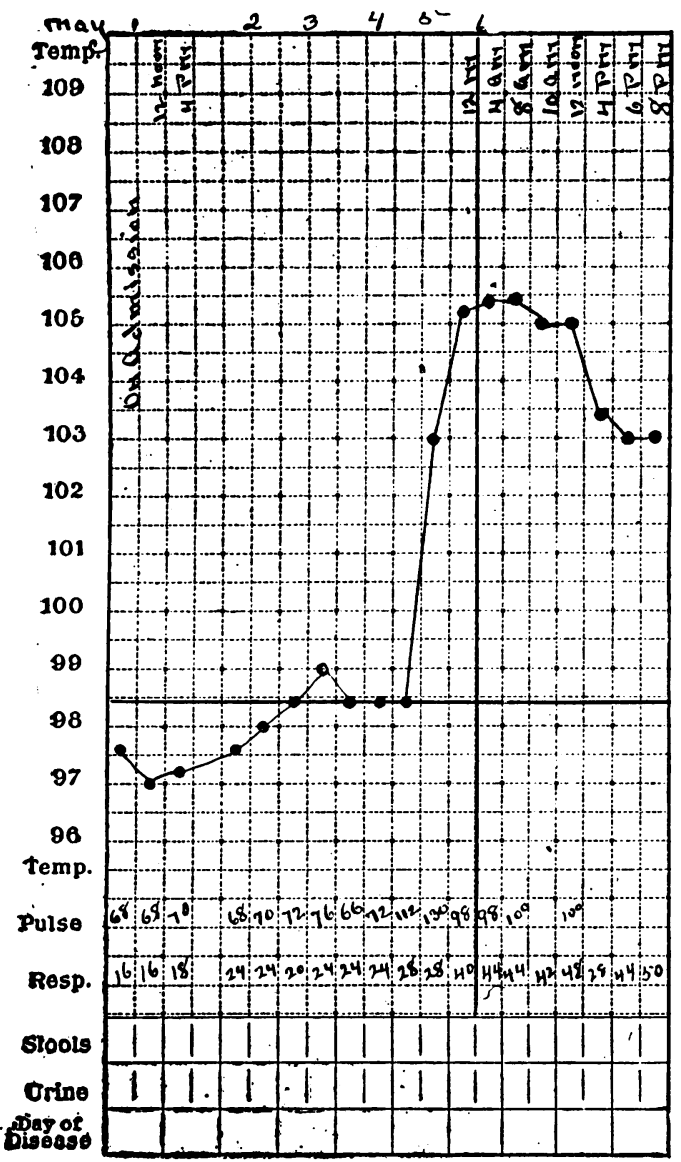

Chart 8, Case I.-P. R.

diplococcus intracellularis of Weichselbaum. but the description of the organism, the position in such numbers within the cells, the failure to grow in cultures and the negative results of experiments on animals, make it more than probable that we were dealing with this organism.

When these cases occurred the disease was not prevailing in Baltimore, and except in New York it was not epidemic anywhere in the country, so far as we could ascertain. Three days after the death of Case I a lad was admitted with a most remarkable family history of the disease. The father, a very intelligent man, gave the following details of the cases:

Wagner Family. - x. A son, a young man of about 20 years of age, returned home on Monday, February 7 th, complaining of a terrible pain in his head. He had fever with much vomiting, and his head and neck were arched. He was very delirious, became rapidly worse, and died on Saturday, February 12th. 2. A sister, who had helped to nurse her brother, was taken ill on Monday, February 14 th, with similar symptoms, and died in four days. 3. A second sister became affected in a few days, and for two weeks was desperately ill ; she then began to improve, and is now well. 4. The mother, who was worn out nursing the children, was taken ill on March 17 th, and died in two days. She had slept in the same

bed with No.5, who was admitted to the hospital. on May r2th, in the eighth week of an illness which the doctor described as cerebro-spinal meningitis, and of which his mother and a brother and sister had died. The patient presented a dull, heavy appearance, and for some days did not appreciate his sur roundings, and appeared confused. He had delusions and hallucinations, and thought he saw his mother and sister in the room with him. He had no fever, and there was no rigidity of the head or neck. The optic nerves looked

I can find no parallel to this record in the history of sporadic cerebro-spinal fever. The figures which I have given for Baltimore show that in the year 1893 a considerably larger number of cases occurred than in the four succeeding years, and the admission of these three cases in rapid succession excited our interest, but from this time until the spring of 1898 we saw nothing further of the disease. In the history of the epidemic form it is not very uncommon to find two or three cases from one house. Netter refers to Sewall's

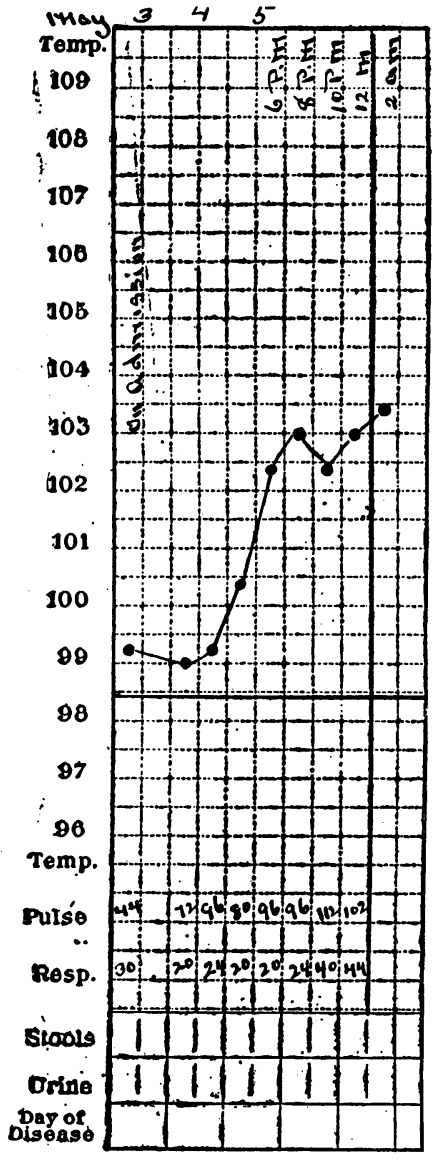

Chart , Case Ir.-W. F.

report of six children attacked in one family at the beginning of the New York epidemic in 1872, and he quotes from Thorne Thorne the statement of Morcieca that in Malta 7 cases occurred in a family of nine persons. Such facts speak strongly for the contagious nature of the disease. Under ordinary circumstances, however, it is exceedingly rare to find more than one case in a family, either in the sporadic or epidemic form, and $I$ know of no case in our recent American experience in which a physician or nurse has been attacked, or in which cases have developed among other patients in a hospital. We know nothing of the conditions under which the disease assumes this family malignancy, as it may be termed. In this respect it resembles pneumonia, not highly contagious as a rule, but which may at times, in a family or institution, present a high degree of malignancy. Bacteriology of Sporadic Cerebro-Spinal Fever.-In a number of
cases of sporadic cerebro-spinal meningitis the Weichselbaum 
organism has been found. The case reported by Stewart and Martin ${ }^{12}$ is of peculiar interest, inasmuch as the epidemic form of the disease has not prevailed in Montreal for many years. It is to be noted, however, that the patient prior to her illness had been spending a few weeks in Boston, where the disease had been prevailing. An unusual point in this case was the occurrence of an acute purulent pericarditis in addition to the usual cerebro-spinal lesions. The cultures showed the diplococcus intracellularis.

By far the most suggestive contribution to this question made of late years is by Dr. George F. Still, ${ }^{13}$ of the Great Ormond Street Hospital for Sick Children. In a study of the form of meningitis known as the simple posterior-basic of infants, he isolated from 7 of 8 cases a diplococcus which conforms in almost every particular with the diplococcus intracellularis. The frequency of this form of meningitis may be gathered from the fact that in ten years there were at this hospital 49 fatal cases, verified by necropsy. The smallest number in any one year was 2, and the largest number 7. All of the cases were sporadic; none of them formed a part of any outbreak which could be considered epidemic. Clinically the disease presents certain differences from the ordinary type of cerebro-spinal fever, attacking young children, chiefly in the first year of life, and as a rule is very much more chronic, though there are forms of cerebro-spinal fever which are quite as protracted. Skin rashes are rare, particularly herpes. The most interesting point of similarity determined by Still is the periarthritic affection in certain of the cases of posterior meningitis, involving, as in epidemic form, the capsule of the joint and the sheaths of the tendons. He determined also in one case the presence of the diplococcus in the exudate.

The problem is one which deserves the closest attention, and in every case of sporadic menirgitis a careful bacteriological examination should be made for the meningococcus and the pneumococcus, as we are still ignorant of the proportion of cases due severally to these organisms. Two points remain for discussion-namely, the incidence of acute nontuberculous meningitis in hospital work, and the clinical varieties of the pneumococcic form.

Through the kind permission of my colleague, Professor Welch, one of his assistants, Dr. W. G. MacCallum, has analysed from the pathological department of the hospital the cases of meningitis in which bacteriological examinations have been made.

The 25 cases may be divided into four groups :

(a) Cerebro-spinal fever-6 cases (Nos. 412, 413, 1,104, 1,189, 1,314 , and 1,362 ).

(b) Pneumococcic meningitis-8 cases (Nos. 384, 478, 619, 746, $989,1,082,1,151$, and 1,362 ).

(c) Pyogenic meningitis, in which streptococci or staphylococci, singly or in association, were found -7 cases (Nos. 694, $1,065,1,092,1,247,1,166,1,363$, and 1,364$)$.

(d) Miscellaneous-4 cases, 2 of which (Nos. 854 and I,09I) showed peculiar unidentified bacilli.

The pyogenic forms of meningitis do not concern us here no case of primary streptococcus or staphylococcus meningitis came to necropsy. I have already referred to the chronic forms of cerebro-spinal fever in which the pyogenic cocci may alone be present at the time of death.

The meningitis with which the diplococcus lanceolatus is associated, in many respects the most important variety, deserves a separate consideration.

\section{Pneumococcic Meningitis.}

As the pneumococcus has long been recognised as the most important organism in the production of meningitis, the first question to be considered is how far sporadie cases of cerebro-spinal meningitis are due to it. Of the 25 cases of meningitis in the pathological department of the Johns Hopkins Hospital, in 8 the pneumococcus was isolated from the exudate. Bacteriological statistic everywhere show the great importance of this organism as a factor in the causation of meningitis. Of 20 cases examined by Councilman, Mallory, and Wright, there were to cases in which the pneumococcus was found, 8 of the se secondary to other conditions, in 2 only primary. In 8 cases of secondary meningitis the streptococcus was present. Netter's experience is particularly interesting. To April, 1897, he had examined bacteriologically 61 cases of meningitis, with the following results: "Pneumococcus in pure culture 35 times, the same associated with the streptococcus and with the staphylococcus each I time, the streptococcus alone 13 times, the diplococcus intracellularis meningitidis 3 times, the staphylococcus pyogenes aureus and the bacillus of Friedlaender each 2 times, and the coli bacillus, the influenza bacillus, a fine bacillus, and a pyocyanic and saprophytic bacillus each I time." We may recognise three groups of cases of pneumococcic meningitis.

I. The Menipgitis is a Complication of Lobar Pneumonia.-In Montreal my attention was called to the great frequency of this association, in 8 of 100 consecutive necropsies, ${ }^{14}$ and since these observations I have had opportunities of studying a. number of cases, though $I$ have never met with so large a proportion. By far the most important contributions to the subject have been those of Nauwerck ${ }^{15}$ and Netter. ${ }^{16}$. Of the 29 cases analysed by Nauwerck, in 4 the convexity of the brain was alone involved, in 16 both cortex and base, and in 17 the cortex, base, and spinal cord. Netter's study, the most complete that has yet appeared, contains an analysis of $124 \mathrm{cases}$ collected from the literature. In the recent monograph on pneumonia by Aufrecht ${ }^{17} 7$ cases of meningitis were found in 253 necropsies, and of 1,501 cases of pneumonia, in 10 the diagnosis of meningitis was made, in 3 of which recovery followed. Of the 8 cases of pneumococcus meningitis in the department of my colleague Welch, in only 4 was the condition associated with lobar pneumonia.

II. Pneumococcic Meningitis from Local Infection.-In this exceedingly important group the infection may.come from the nose or the adjacent sinuses, from the ears, or may follow an injury to the skull or an operation. In 2 of the 3 cases in the series analysed by Dr. MacCallum there was fracture of the base of the skull. In the third, a child, aged 6, had suppuration of the nasal mucous membrane and adjacent sinuses. In this connection the importance of the pneumococcus is universally recognised, and many cases are on record of infection from an otitis media.

III. Primary Preumococcic Meningitis.-A number of observers have isolated the pneumococcus in primary suppurative meningitis. The records cannot be regarded, however, as entirely trustworthy, since it is only within the past few years that bacteriologists have learned to recognise the differences between the pneumococcus and the diplococcus intracellularis. Subsequent study must determine in what proportion of cases of sporadic meningitis the pneumococcus exists, and in how many the diplococcus intracellularis.

The primary pneumococcic meningitis may develop in a person in good health, but more frequently there is a general pneumococcus infection in a debilitated individual, or in one with chronic disease. The meningitis may be present alone, or as is so common, in association with endocarditis. Of the cases examined in the post-mortem room of the Johns Hopkins Hospital, one (No. 989), a man aged 44, had a general infection with the pneumococcus and meningitis. Another case (No. 1,206), with a general infection; acute endocarditis, and meningitis, is not included in this series, as a vartial necropsy only was allowed, and but a small portion of the lumbar cord was examined.

Clinical Features of Pneumococcic Meningitis.-I shall now refer to certain clinical features of these three groups of cases :-

(a) Pneumonia with Complicating Meningitis. - Is the case one of cerebro-spinal fever with pneumonia or of inflammation of the lungs with an added meningitis? This question does not often arise now at the bedside, as it is most exceptional for the meningitis of pneumonia to present the symptoms of cerebro-spinal fever and in a dubious case occurring during an epidemic the lumbar puncture (as in a case to be referred to in a few minutes) may be relied upon to clear up any doubts. The most valuable clinical record of meningitis in pneumonia is to be found in Nauwerck's paper. The histories, 17 in number, are very full and complete, and in every case accompanied with a post-mortem report. He has added $a$ series of 12 cases from the literature, making 29 in all. All of the cases were above the 2oth year of age-a striking contrast to cerebro-spinal fever, in which a large proportion of all the cases are in the young. A second point is the latency of meningitis in pneumonia, which is much more often recognised in the dead house than in the wards. Netter states that fully one-half of the cases are of .this latent type. 
Of the cases I saw in Montreal I remember but one in which the diagnosis was made during life. Huguenin, referring to this obscurity, says: "We know no symptom which is constant in all cases, or which may not be present in an identical manner in other conditions." Headache, early delirium, deepening into unconsciousness, are present in all cases. This is a consequence of the more common involvement of the cortex of the hemispheres. As Ieichtenstern remarks, the mind may remain clear throughout the course of a case of cerebro-spinal fever. Spinal symptoms are rare; in only 7 of the cases analysed by Nauwerck was there rigidity of the neck muscles. Strabismus was present in one-fifth of the cases, ptosis only once. The importance of lumbar puncture cannot be too strongly emphasised. A recent case, which occurred while cases of cerebro-spinal fever were in the hcspital, illustrates this so well that I give an abstract of it :

C. E., aged 52 (Hospital No. 26417), was admitted May 6th, 189o. with pneumonia of the right upper lobe of about eight days' duration. He was in a semi-comatose condition, with a temperature of only $99^{\circ}$. respirations $3^{2}$, pulse ro8. There was a pneumonia of the apex of the right lung. The day after admission it was noted that his arms and legs were very stiff; The patient, however, responded to questions. Lumbar puncture was made in the third interspace, and $5 \mathrm{c.cm}$. of a perfectly clear limpid fluid obtained. Lumbar puncture was made again on May roth, and $30 \mathrm{c.cm}$ of a perfectly clear fluid obtained, negative on cover-slip and culture For the next three days he had a good deal of tremor. Kernig's sign was present, and there was still some rigidity of the arms and of the neck. The leucocytes ranged from 16,000 to 24,000 per c.cm. On the 14 th lumbar puncture was performed for a third time, and a slightly turbid fluid obtained, from which on cover-slips the pneumococcus was obtained. The patient died on the evening of May 14 th. The necropsy showed a pneumonia of the right apex, a very slight purulent exudate at the base. some turbid fluid in the ventricles, and a small amount of turbid cerebrospinal fluid, but no positive exudate on the spinal meninges. There was an ulcerative endocarditis of the aortic valve. The pneumococcus was isolated from the meninges, the heart valves, and from the lung. A very interesting festure in this case was the normal temperature on the
eleventh. twelfth. and thirteentl days of the disease, and the high temperature on the fourteentl and fifteenth days, ranging to $105^{\circ}$ and

And lastly, a most important difference between the meningitis complicating pneumonia and cerebro-spinal fever is the almost universally fatal course of the former. Nauwerck describes the complication as invariably fatal, and he was not able to convince himself that in any one of the reported cases of recovery in the literature meningitis was actually present. Netter $^{18}$ speaks somewhat more hopefully, and states that recovery may occur. He gives two cases from the literature, both in children. In one, a child of 5 , in which the crisis occurred on the sixth day, the signs of meningitis were the presence during the first few days of headache, unequal pupils, and ptosis. Aufrecht, in his recent monograph (loc. cit.), also speaks of the possibility of recovery. Of 10 cases of meningitis in 1,5 or of pneumonia, 3 recovered. Netter, in his article in the Iiventieth Century Practice, commenting upon the statement by Wentworth that pneumococcal meningitis is always fatal, states that "as early as 1887 I demonstrated. however, that its curability is one of the most evident characteristics of the pneumococcal meningitis." So far as I gather there is nothing in his essay to justify such a statement, since, as I have mentioned, he gives but two instances of recovery.

Personally I have never recognised recovery in pneumonia complicated with meningitis, and the literature of the subject bears out strongly the view that one of the most striking differences between the meningitis of pneumonia and cerebrospinal fever is the almost invariably fatal termination of the former.

(b) Pneumococcic Meningitis from Local Infection.-Two of the three cases in this group were surgical ; one (No. 746), followed removal of the bones of nose and orbit; the other $(1,151)$ a fracture of the base of the skull, involving the cribriform plate of the ethmoid. In both the pneumococcus alone was isolated from the exudate in the cerebral meningitis. In 2 of Councilman's 8 cases the infection followed fracture, in I otitis media. Oases of this group do not so often appear in the medical wards, but last year we were particularly interested in a case in which the infection started from the nose and accessory sinuses; though the possibility has to be considered of extension from the meninges. An abstract is worth quoting, and the picture (Fig. 2) shows a remarkable condition of cervical opisthotonos:

A. C. ared 6 (black), admitted March 3rst, ${ }^{2808}$. Her previous history was negative. Five days before entrance, directly after supper, she vomited and complained of headache; later in the evening she became delirious. Two days after the head was held stiffly, and she had much pain in the back of the neck. She had fever, rapid pulse, loss of appetite, and constipation. On admission she was conscious, but very irritahle and difficult to examine. The head was arched back and could not be brought forward. The pupils were equal and reacted to light. There was a marked slight internal strabismus. There was nothing of moment found in the examination of the chest and abdomen. She was very tender in the suboccipital region, but there was no sign of disease of the cervical spine. Lumbar puncture on April ist was negative. For the first week the condition remained about the same; the child could be roused and answered questions: the strabismus persisted : there were no changes in the optic nerves. The temperature ranged from $100^{\circ}$ to $104^{\circ}$. The arching of the head and neck continued; the eyes looked directjy up wards (Fig. 2). On April 17 th a rash appeared, a diffuse erythema over the ace, neck, and chest, with papules. The rash faded in three days. The temperature ranged from ro2 ${ }^{\circ}$ to $104^{\circ}$. On April roth a profuse purulent discharge appeared from the nose which showed the pneumococcus on cover slips, but in cultures only the staphylococcus aureus grew. She could still be roused the attitude remained as shown in the photograph. No optic neuritis. Throughout the 18 th the temperature dropped from ro $5^{\circ}$ to $97^{\circ}$ on the morning of the 19 thil, and continued about $97^{\circ}$ until her deth ou the moruing of the 2 sst. One of the most remarkable
was the retention of consciousuess to within a wezk of her death.

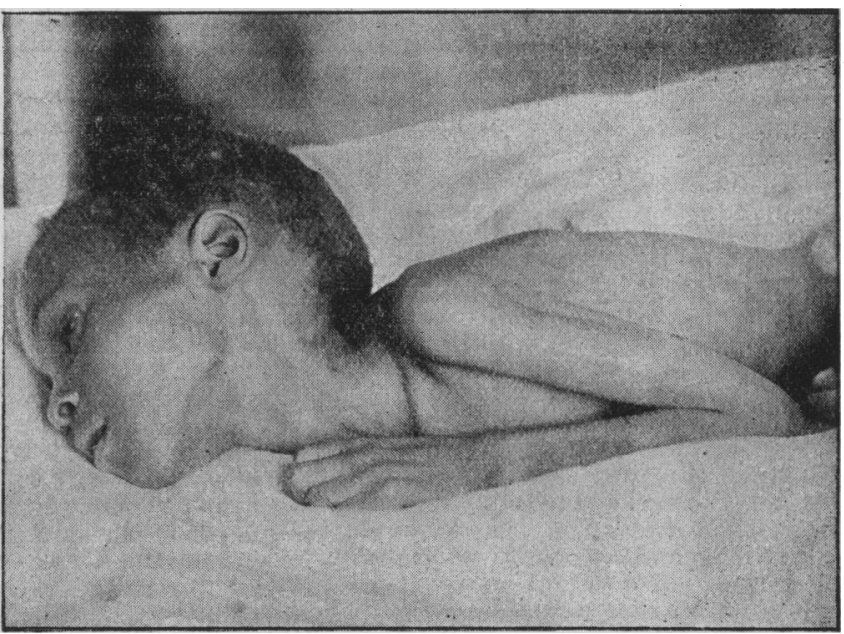

Fig. 2.-A. C. Pneumococcic meningitis-cervical opisthotonos.

The necropsy (No. r,082) showed exudate over the sulci of the hemi spheres. The base, from just behind the optic commissure, over the pons and lateral lobes of the cerebellum, waph cover exudate. The posterior surface or the spinal everd sh whe entrict tion of the mucous membrane of the hes and of the ethoidal and spinal exudate, and from the nasal pus the pneumococcus and the staphy(c) Primary $P$

(c) Primary Pneumocnccic Meningitis.-Future investigations will decide the proportion of cases of primary cerebro-spinal meningitis due to the pneumococcus and to the diplococcus intracellularis; and it will be an exceedingly interesting study to determine whether there are clinical differences such as separate so decidedly the meningitis complicating pneumonis from the cerebro-spinal fever. Judging fron the bacteriological records given by Councilman, Mallory, and Wright, and those of the Pathological Laboratory of the Johns Hopkins Hospital, the primary pneumococcic meningitis is rare in America-certainly much less infrequent than the secondary forms. Two of Councilman's 10 cases, both infants, one of ten months, the other of six days, were primary. I cannot gather from Netter's statistics 19 how many of his cases were primary ; nor without consulting the original can one say how many of the 13 cases of sporadic meningitis studied by Malenchini (quoted by Netter) belonged to this group. Of our 8 cases, as stated, only I was primary, and is worth quoting

G. C., aged 44 (white), admitted at 6.30 P.M. on September 1 st, 1897 , in an unconscious state. When seen by his brother on Saturday, August 29 th, he was quite well. Aftergoing to bed on Saturday evening, the 3oth, he had a severe chill. On Monday he was too ill to go to work. In the evening he became delirious, and ever since has had fever, has been restless, difficult to restrain, and very noisy. On admission the temperature was ro $5^{\circ}$; the pulse 136 and small. Dr. McCrae saw him a few minutes after he had heen put to bed, and took very full and careful notes of the condition. The face was fushed and he looked distressed. There was no strabismus. the pupils were dilated and inactive. The head and neck were held signs of pneumonia ; the heart sounds were clear, The abdomen was not
s. 
distended, there were no rose spots, and the spleen could not be felt; there were no herpes. The skin had a diffuse mottling, but no purpuric spots were seen. There was no urethral discharge. The leucocytes were 13,500 per c.cm., no alteration in a differential count of 500 . The Widal re-
action was not present. At ro P.M. the temperature had reached 106 $6^{\circ}$ and he was given a bath at $80^{\circ}$, with ice frictions, which reduced the rectal he was given a bath at $80^{\circ}$, with ice frictions, which reduced the rectal stiffly. At 12 midnight he was much worse, and at 12.40 A.M. he died, about stiffy. At 12 midnight he was much worse, and at $12.40 \mathrm{~A}$.M. he

The post-mortem examination (No. 939) showed a diffuse purulent meningThe post-mortem examination (No. 939) showed a diffuse purulent mening itis of the hemispheres, the base much less involved and the ventricles not at all affected. The cord unfortunately was not examined. There was and men and tube casts. There was a general pneumococcus infection, no culture from the meningeal exudate.

The Treatment of Cerebro-spinal Fever.

In our series of cases we have used no special drugs. Morphine has been freely given to control the pain. Ice sponging has been employed whenever the temperature reached above $102.5^{\circ}$. Our mortality, considering that the cases were as a rule quite severe, has been low-only 8 of 18 cases in the hospital, and 9 among the 21 cases which $I$ hrve seen.

I have already spoken of the possible benefits in certain cases of the relief of pressure by the withdrawal of cerebrospinal fluid.

In two of our cases the spinal canal has been opened, drained and irrigated. Winter ${ }^{20}$ in tuberculous meningitis, removed the final process of the second lumbar vertebra and drained the spinal canal. Von Ziemssen first attempted to use local therapeutics to the membranes of the cord by injecting a weak solution of iodine in a case of meningitis; and later Sahli practised permanent drainage through a cannula and catheter. - So far as I know, an extensive laminectomy had not been done for acute spinal meningitis until our first case, November 6th, 1898, in which the operation was suggested and performed by. Dr. Harvey W. Cushing the first assistant in the surgical clinic of my colleague, Professor Halsted. The case has already been given in abstract under the section on Bacteriology ${ }^{21}$ as the one in which the staphylococci only were present at the time of operation. The spinal canal was thoroughly irrigated with salt solution and a quantity of purulent exudate washed out. No change followed in the existing paraplegia. The bladder and kidneys became infected, and he died about two months after the operation. The paraplegia persisted. At necropsy the spinal meninges were smooth and looked perfectly normal. It was impossible to say where the dura mater had been incised, and there were neither adhesions nor areas of the thickening on the pia arachnoid. There were extensive changes in the cord itself and in nerve roots.

In Case XII laminectomy was performed on the fourth day by Dr. Cushing. The patient was very ill, and the spinal symptoms were especially propounced. A catheter was passed beneath the dura mater, and the membranes drained and irrigated with salt solution. For several days he seemed much better. He developed a hæmorrhagic cystitis and pyelonephritis, and died on the sixth day after operation.

Dr. Musser, of Philadelphia, has also had an unsuccessful case. Dr. Rolleston and Mr. Herbert Allingham have reported ${ }^{22} a$ case of sporadic cerebro-spinal meningitis, in which the patient recovered after laminectomy and drainage. On the principle of a desperate remedy for a desperate disease, the operation (which has been criticised adversely in an editorial note in the Philadelphia Medical Journal) seems justifiable in certain severe cases, in which, as in our first case, the spinal symptoms are very marked.

' NOTE AND REFERENCES.

1 Public Health Reports, vol. xiv. 2 "Epidemic Cerebro-spinal Menigitis." A Report of the State Board of Health of Massachusetts, Boston, 1898 . 3 Jahr. f. Kinderheilk., 1891, and the Deut. med. Woch., 1897. 4 Lnc. cit.. P. 193. Bull. et Mém. Soc. Méd. des Hôp. de Paris, xv, p. 407,1898 . The case of John Brooks (Necrops, No. I, o65) is of interest in this connection. He had an illness of eight weeks' duration, with obscure cerebral symptoms. On admission he had a urethral discharge. At necropsy there was found an extensive cerebral meningitis, with distension of the ventricles and slight meningitis of the dorsal cord. From the exudate and from the urethral discharge streptococci and staphylococci were isolated. The slight urethral secretion might readily have been overlooked, and the case regarded as a primary pyogenic meningitis. 7A full report of these cases is given in the Boston Medical and Surgical Journal, December

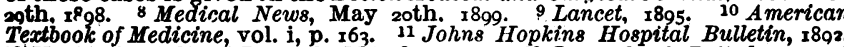
T12xtbontreal Medical Journal, March, 1898. 13 Journal of Pathology and
Bacteriology, vol. v, 1898. 14 The Morbid Anatomy of Pneumonia, Canada Medical and Surgical Journal, May, 1885 ; and the Gulstonian Lectures on Malignant Endocarditis, BRITISH MEDICAL JOURNAL, 1885, i; 15 Deut.

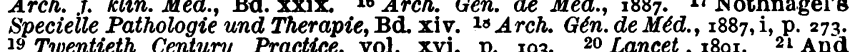
reported in Bozton Medical and Surgical Journal, December 29th, 1898. ${ }_{22}$ Lancet, $x 899$.

\section{THE CROONIAN LECTURES}

ON

\section{SOME POINTS CONNECTED WITH SLEEP, SLEEPLESSNESS, AND HYPNOTICS.}

Delivered before the Royal. College of Physicians of London. BY JOHN BUCKLEY BRADBURY, M.D.CANTAB.,

Fellow of the College; Downing Professor of Medicine in the University of Cambridge; and Senior Physician to Addenbrooke's Hospital.

\section{LECTURE I.}

WHEN the late President did me the great honour of asking me to deliver the Croonian Lectures for 1899, I felt some diffidence in accepting the invitation; but on thinking the matter over it occurred to me that some points connected with sleep, sleeplessness, and hypnotics, which had engaged my attention, might possibly be of sufficient interest to bring before the College. Let me therefcre thank the College for the task which was entrusted to me, and ask you to consider with me, in the first place, the

Physiology of Sleep.

For a long time sleep has been viewed from two standpoints - the physiological and the psychological-and although an attempt to combine the two is sometimes observed, they have remained distinct to the present day; for, notwithstanding the great development of physiological psychology, little has yet been done to bridge over the great gulf between the two sciences. But tine question of the correlation of physiology and psychology may yet be solved, and the solution is necessary for a true conception of the phenomena of sleep.

On the basis of recent discoveries in the physiology, and more particularly in the histology, of the nervous system, but before we can consider these in detail it is necessary to review our present conception of the central nervous system. The view now current is based upon Waldeyer's idea of a nervous system made up of independent elements-neurons and other cellsintimately and complexly arranged. Each neuron consists of a cell body with branching processes (dendrons or dendrites) and an axis cylinder process (axon or axite). The dendrons springing from various parts of the cell body divide and subdivide until extremely fine terminal processes (dendrites) are reached, which end apparently freely in a homogeneous matrix. In many cases upon the dendrites short lateral offshoots termed gemmules or thorns may be seen, and occasionally, especially in the young, nodosities may also be noted. The axon, contrary to former belief, is known to give off branches or collaterals, which again subdivide among the dendritic processes of other cells or form a network around tinuity. Each neuron is an independent unit, and as such comparable with the cells forming other organs of the body. The supposed mode of combination of the various nerve cells is shown in the diagram on p. 1528, col. r, taken from Ramón y Cajal's Croonian Lecture.

The neurons, as might be expected, vary in the different parts of the brain and cord, both in regard to the size, form, and intimate structure of the cell body, and to the form and branching of its processes. But our immediate purpose is rather with the function than with the size and form of the cell. Those anatomical features that are concerned with the function of the cell are alone in the present connection of interest, and latterly they have received considerable attention. It is said that the fibrils of the axon process can be traced in some cells into the dendrons, and it has been stated that the function of the cell body, in such cases, is purely nutritive. Generally in the cell body a granular formation, more or less definitely arranged, is observed, the granules around the nucleus being more or less spherical, those at the perjphery more elongated. These granules are believed to be connected with the functional activity of the cell ; they disappear, for example, from the cells of the cervical ganglion after long-continued electrical stimulation (Vas, Lambert. Hodge, and the cells of the cerebral cortex, even when affected by ordinary stimuli, show a diminution in their staining power when compared with unstimulated cells (Mann, Lugaro, Demoor). Changes in the form and volume of the cell have also been described. With moderate activity, turgescence of the cell and nucleus and accummulation of chromatin particles occurs, and after prolonged work shrinking of the protoplasm and

All physiologists who accept Waldeyer's view believe that the transmission of nerve impulses occurs through the intercalated material between the terminal cell processes, but 